\title{
OBSERVATIONAL PROPERTIES OF COMPACT GROUPS OF GALAXIES
}

\author{
Paul Hickson \\ Department of Geophysics and Astronomy \\ University of British Columbia
}

\section{Introduction}

Compact groups are small, relatively isolated, systems of galaxies with projected separations comparable to the diameters of the galaxies themselves. Two well-known examples are Stephan's Quintet (Stephan, 1877) and Seyfert's Sextet (Seyfert 1948a,b). In groups such as these, the apparent space density of galaxies approaches $10^{6} \mathrm{Mpc}^{-3}$, denser even than the cores of rich clusters. The apparent unlikeliness of the chance occurance of such tight groupings lead Ambartsumyan $(1958,1975)$ to conclude that compact groups must be physically dense systems. This view is supported by clear signs of galaxy interactions that are seen in many groups. Spectroscopic observations reveal that typical relative velocities of galaxies in the groups are comparable to their internal stellar velocities. This should be conducive to strong gravitational interactions - more so than in rich clusters, where galaxy velocities are typically much higher. This suggests that compact groups could be excellent "laboratories" in which to study galaxy interactions and their effects.

Because of their small sizes, compact groups have relatively short dynamical times. The orbital times of galaxies in such groups is of order a tenth of the Hubble time. It seems unlikely that the densest groups could avoid destruction by galaxy mergers for the age of the universe. They must therefore be young systems. We are thus faced with the related questions of how they form, and what becomes of them. If, as suggested by numerical simulations, the merger products resemble elliptical galaxies, what fraction of present day ellipticals were made in compact groups?

A complication to this picture is the amount and extent of dark matter in compact groups. It has been known since the work of Burbidge and Sargent (1971) that the velocity dispersions of many compact groups are larger than expected if the only mass in the groups is that associated with the galaxies. Mass to light ratios inferred from the virial theorem are often larger than those of individual galaxies by as much as an order of magnitude. Either the groups are not in equilibrium, or the majority of mass in the group is in an unseen form, possibly as a smooth envelope through which the galaxies move.

Compact groups often contain one or more galaxies whose redshift differs greatly from those of the other group members. If these galaxies are at the same distance as the other members, either entire galaxies are being ejected at high velocities from these groups, or some new physical phenomena must be occurring. If their redshifts are cosmological, we must explain why so many discordant galaxies are found in compact groups. 
In recent years much progress has been made in addressing these questions. In this review I discuss the current observational data on compact groups and their implications. The dynamical theory of dense groups of galaxies has been reviewed by Barnes (1990), and by Mamon in these proceedings. The reader is also referred to the review by White (1990) of apparent paradoxes posed by compact groups. Effects of the environment on galaxies in clusters and compact groups have recently been reviewed by Whitmore (1990).

\section{Catalogs}

The Palomar Observatory Sky Survey (POSS), completed almost forty years ago, provided a unique database for studies of compact groups of galaxies. The first catalog containing compact groups, the "Atlas of Interacting Galaxies" (Vorontsov-Vel'yaminov 1959, 1977 hereafter VV) contains galaxies and small groups selected on the basis of visible signs of interaction on the POSS prints. Although group membership was not a selection criterion, this catalog contains Sephan's Quintet and Seyfert's Sextet and similar groups such as the famous galaxy chain VV172. The catalog also contains about 200 objects referred to as "nests" by Vorontsov-Vel'yaminov (eg. VV644, VV645) which look like small groups of very blue galaxies in physical contact. Subsequent studies of several VV nests (Barbieri et al 1979, Arkhipova 1982, Arkhipova et al 1987, Vorontsov-Vel'yaminov, Dostal', and Metlov 1980) have shown that many of these nests are in fact single irregular galaxies. The Arp "Atlas of Peculiar Galaxies" (Arp 1966), contains galaxies selected on the basis of peculiar appearance on the POSS prints. It contains a number of small groups, many of which are compact, including many of the Vorontsov-Vel'yaminov compact groups as well as several new systems. The high-resolution photographs in this atlas clearly illustrate the kinds of interaction seen in many compact groups.

In 1957, Shakhbazyan discovered a remarkable group of 12 faint galaxies which appear so compact that they were originally mistaken for stars (Shakhbazyan 1957). Subsequent investigations of Shakhbazyan 1 (Robinson and Wampler 1973, Arp, Burbidge and Jones 1973, Beekman 1974, Klimek 1974, Thompson 1976, Massey 1977, Shakhbazyan 1978, Kirshner and Malumuth 1980) revealed it to be a small cluster of luminous red compact elliptical galaxies with a recession velocity of $33000 \mathrm{~km} \mathrm{~s}^{-1}$ and a velocity dispersion of only $62 \mathrm{~km}$ $\mathrm{s}^{-1}$. Over a period of several years, Shakhbazyan and collaborators surveyed over 200 POSS prints, covering about $18 \%$ of the sky, in search of similar groups. They used three selection criteria:

There must be at least 5 members, but large clusters are excluded. Most members must appear compact on either the red or blue print. The group itself must be sufficiently isolated or compact.

The results were published in 10 lists containing a total of 377 "compact groups of compact galaxies" (Shakhbazyan 1973, Shakhbazyan and Petrosyan 1974, Baier et al 1974, Petrosyan 1974, 1978, Baier and Tiersch 1975, 1976a,b, 1978, 1979, hereafter CGCG). Some general properties of 305 CGCG were reviewed by Tiersch (1976). Most of these appear to be groups of red compact galaxies. They have a wide range of densities and velocity 
dispersions. This sample of groups contains many interesting objects which have been the subject of detailed studies (Börngen and Kalloglyan 1974, Mirzoyan, Miller and Osterbrock 1975, Shakhbazyan and Amirkhanyan 1978, Amirkhanyan and Egikyan 1987, Kodaira, Iye Okamura and Stockton 1988).

The first large statistical study of compact groups was published by Rose (1977) who examined 69 blue POSS plates and also 23 plates from the Yale-Columbia survey (Wesselink 1974) covering a total area of about $7.5 \%$ of the sky. Rose used two quantitative selection criteria,

$$
\begin{aligned}
& n \geq 3 \text { with } m \leq 17.5 \\
& \sigma_{f} A_{G} \leq 0.0035
\end{aligned}
$$

where $n$ is the number of members, $m$ the estimated galaxy magnitude, $\sigma_{f}$ the average surface density of field galaxies, and $A_{G}$ the area of sky covered by the group. The second criterion corresponds to requiring the group to have a surface density enhancement of about a factor of 1000 over the field. Rose found 170 galaxy triplets, 33 quartets and 2 quintets obeying these criteria. The typical magnitude spread between galaxies in a group is small, less than about 1.5. The typical area of a group is less than one square arcmin. Rose also obtained photographic photometry for 2 groups, in order to look for diffuse background light from stars stripped from the galaxies by gravitational interactions (Rose 1979).

The most recent, and largest, survey of compact groups is that of Hickson (1982) who visually inspected all the red POSS prints, covering about $67 \%$ of the sky. Three selection criteria were used

$$
\begin{aligned}
& n \geq 4 \text { with } m \leq m_{B}+3 \\
& R_{N} \geq 3 R_{G} \\
& \bar{\mu}_{G}<26
\end{aligned}
$$

where $m_{B}$ is the estimated red magnitude of the brightest group member, $R_{G}$ is the radius of the smallest circle containing the centers of the group members, $\bar{\mu}_{G}$ is the mean surface brightness contained by this circle and $R_{N}$ is the distance from the center of the circle to the nearest nonmember galaxy satisfying the same magnitude criterion. Exactly 100 groups were found which satisfied all three criteria. Some minor changes to the membership of the original catalog were made by Hickson, Kindl and Auman (1989), to ensure compliance with the selection criteria using photometric magnitudes derived from CCD images. The catalog contains 60 quartets, 25 quintets, 8 sextets, 6 septets and one octet. These include the classical compact groups, several VV, Arp, CGCG and Rose groups plus a number of new groups. These groups have apparent space densities spanning a range of over four orders of magnitude, including the highest density systems known. The faintest galaxies in the sample have blue magnitudes of 19 to 20 .

This sample has received extensive observational study. Optical spectra and redshifts have now been obtained for nearly all galaxies (Hickson et al 1984, Tikhonov 1986, Hickson, 
Kindl and Huchra 1988b, Hickson and Huchra, 1990, and work in progress). 6-m photographic plates have been obtained for all groups (Tikhonov 1987a,b, 1989), and broad-band (B and R) CCD images have been obtained for all galaxies (Hickson, Kindl and Auman 1989) magnitudes and Hubble types have been derived from both these data sets. Radio studies include HI line observations (Williams and Rood 1987, Williams and van Gorkum 1988) and continuum observations (Menon and Hickson 1985). Infrared (Hickson et al) studies based on the IRAS data base have now been published, and X-ray observations exist for several groups (Bahcall, Harris, and Rood 1984). In addition, there have been several detailed studies of the neighborhoods of these compact groups (Sulentic 1987, Rood and Williams 1989, Kindl 1990).

Hickson's catalog is now perhaps the best studied sample of compact groups, and much of the discussion that follows in this review will be based on it.

\section{Spatial Distribution and Environment}

The environment of compact groups provides important clues to their nature. Are they truly isolated, or are they associated with large clusters, or with looser groups of galaxies? A cursory examination reveals that Hickson's compact groups (hereafter HCGs) are quite uniformly distributed on the sky (away from the galactic plane). They do not concentrate toward the supergalactic equator, nor do they show any clear preference for identifiable clusters or clouds of galaxies with comparable redshifts. In a careful study of the environment of compact groups, Sulentic (1987) finds that only 8 HCGs are in or near rich clusters or groups.

A more difficult question is whether the groups are associated with poor groups, or with other galaxies in their vicinity. Rose (1977) concluded that most of his groups appear to be surrounded by a "halo" of one or more galaxies with a typical radius of 5 to 10 group radii. Sulentic (1987) examined all galaxies within $1^{\circ}$ of all HCGs and concluded that the average surface density of galaxies increases slightly within $0^{\circ} .5$ of a group, where it is comparable to that of the lowest density loose groups. He found that typical HCGs have density enhancements of a factor of 100 or more, and about a third have density enhancements of over 500. Rood and Williams (1989) examined galaxies within 10 group radii of the HCGs, and found no significant excess of galaxies in the neighborhoods of two thirds of them. They estimated that on the average there is one physical neighbor for every 3.3 group members, which seems to be in accord with the findings of Rose. Kindl (1990) examined all galaxies within $1.125 \mathrm{~h}^{-1} \mathrm{Mpc}$. of $97 \mathrm{HCGs}$ with known redshifts, and estimated their magnitudes and Hubble types. ( $\mathrm{h}$ is the Hubble constant in units of $100 \mathrm{~km} \mathrm{~s}^{-1} \mathrm{Mpc}^{-1}$.) This is the largest environment survey, comprising almost 4000 galaxies. He finds that $78 \%$ of the groups show no significant excess of galaxies within $0.5 \mathrm{~h}^{-1} \mathrm{Mpc}$. Kindl confirms the conclusion by Rood and Williams that the HCGs have a lower spiral fraction than their neighborhoods, and that this difference is more pronounced for groups with a significant excess of nearby galaxies. This result is of particular significance in indicating that compact groups are physically distinct from their environments.

These three studies agree that most HCGs are truly isolated, showing no significant 
excess of galaxies in their vicinity. However, about one in five groups do appear to be associated with larger systems, typically poor groups. There is evidence that the fraction of spirals in the groups is smaller than in their environments, particularly for the groups associated with larger systems.

\section{Morphology}

Since the HCGs form a relatively large, homogeneous sample, it is possible to draw general conclusions about their average properties. At this stage it will be useful to summarize the principal observational results:

a) optical

Many galaxies in compact groups are interacting. It is estimated that roughly one third of the galaxies in HCGs show clear signs of interaction, such as tidal distortion or truncation in the imaging data alone (Palumbo et al 1990). When spectroscopic data are included, the number may be even higher. Fully two thirds of the 33 spiral galaxies observed by Rubin, Hunter and Ford (1990) have peculiar rotation curves. Galaxies that are not now interacting may have suffered past interactions or mergers. Zepf and Whitmore (these proceedings) have confirmed that several of the elliptical galaxies in the HCGs have unusually blue colors that may have resulted from a recent burst of star formation.

The morphological types of galaxies in the HCGs show several interesting trends. The fraction of spirals in the groups averages about $50 \%$. This fraction correlates weakly with group density (see the discussion in Whitmore 1990), more strongly with group luminosity, and most strongly with group velocity dispersion (Hickson, Kindl and Huchra 1988b). Groups with high velocity dispersion contain few spirals. This seems to be opposite to what would be expected if elliptical galaxies are being produced by dynamical evolution. Gravitational interactions and mergers should proceed more rapidly in groups with low velocity dispersions as slow encounters are more effective in disrupting galaxies. Hickson, Kindl and Auman (1989) conclude that the morphology - velocity dispersion correlation is most likely due to environmental effects at the time of galaxy formation.

A related correlation first noted by Sulentic (1987) is that of morphology concordance. Galaxies within a group are more likely to have the same morphological type (spiral or nonspiral) than a random sample. White (1990) has pointed out that such a correlation is expected as a consequence of the morphology-velocity dispersion relation. It should be possible, by numerical calculations, to determine whether this effect is strong enough to explain the observed degree of concordance.

It is generally agreed that mergers should produce elliptical galaxies, and so it was expected that the first ranked galaxies in groups should have a lower spiral fraction than the other galaxies. It was therefore a surprise when Hickson (1982) (also Hickson, Kindl and Huchra 1988b) found no significant difference between the morphological type of the first ranked galaxy and the others. Whitmore (1990), however has argued that this can be explained by the binning used in these studies. If $\mathrm{S} 0$ galaxies are grouped with spirals rather 
than ellipticals, on the grounds that they are not expected to be produced by mergers, a significant correlation does emerge. This suggests that some merging has already occurred in many groups.

Preliminary studies of the optical luminosity function of galaxies in compact groups indicate that it is similar to that of field galaxies (Hickson et al 1984). Using the completeness corrections of Hickson, Kindl and Auman (1988), Hickson (1989, unpublished) estimates the luminosity density of all galaxies in compact groups to be of order $2 \times 10^{6} \mathrm{~h} \mathrm{~L}_{\odot} \mathrm{Mpc}^{-3}$. Using the estimate $3 \times 10^{8} \mathrm{~h} \mathrm{~L} \odot \mathrm{Mpc}^{-3}$ for the luminosity density of all galaxies (Bingelli, Sandage and Tamman 1988), he concludes that compact groups contain almost $1 \%$ of the light of the total galaxy population. Compact groups contain a significant fraction of the total light of galaxies in the universe. Although the overall luminosity function may be normal, within individual groups, their are deviations. Heiligman and Turner (1980), Hickson (1982) both report evidence for a deficiency of fainter galaxies, compared to a normal luminosity function. For the HCGs this may be due, at least in part, to the selection criteria, which encourages luminosity concordance. It remains to be seen whether this can account for the observed anomalies. If the effect is real, it would indicate that mass segregation is an important aspect in the formation or evolution of compact groups.

The shapes of compact groups have been investigated by Turner and Sargent (1974), Rose (1977), Hickson et al (1984), Malyck and Orlov (1986). The first two studies concluded that the observed elongations of compact groups were consistent with a random distribution of galaxies. The latter studies examined the HCGs and concluded that the groups are more elongated than a random distribution, and cannot therefore be explained by chance superpositions of galaxies. The data indicate that the groups are best represented by intrinsically prolate spheroids. Of course the shape of any small dense group will change significantly in a crossing time (the time taken for a typical galaxy to move a distance equal to the radius of the group). Prolate average shapes could arise from radial galaxy orbits, or outlying members in circular orbits.

b) radio

Hydrogen line radio observations have been made of spiral galaxies in 51 HCGs by Williams and Rood (1987). They find that these spirals have generally only about half as much neutral hydrogen as a comparable sample of isolated galaxies. They also find that about a third of these galaxies have distorted line profiles. Higher-resolution studies (Williams and van Gorkom, 1988, Williams, McMahon and, van Gorkom, these proceedings) show the HI emission encompassing the whole group in three of the four groups studied.

Menon and Hickson (1985), have observed $88 \mathrm{HGC}$ with the VLA at a wavelength of $20 \mathrm{~cm}$. Many of these have subsequently been observed at $6 \mathrm{~cm}$. 41 sources were found above a flux limit of $1.5 \mathrm{mJy}$. In the spiral radio galaxies, emission is seen predominantly from the circumnuclear regions, but in elliptical galaxies, only nuclear emission is seen. These radio ellipticals are almost always first-ranked optically, but surprisingly, the optical luminosity of the galaxy is not strongly correlated with the radio emission. The relative rank of a galaxy seems to be more important than its luminosity in determining the frequency of radio emission. This result suggests that the radio emission is related to the galaxy's location or prominence in a group. Precisely the same effect has been independently found 
by Tovmasyan and Shakhbazyan (1981) in loose groups and double galaxies.

Another interesting feature of the radio observations is the almost total absence of extended radio emission. The observed flux is contained mostly within the visible galaxy. No extended sources such as the large double-lobed or head-tail sources that are seen in clusters are present. Either the physical processes in these galaxies are different or the activity has started only recently and extended components have not had time to develop.

c) infrared

In a recent study based on the IRAS data base, Hickson et al (1989) conclude that the far infrared emission of galaxies in HCGs is enhanced by about a factor of two compared to a sample of isolated galaxies. The infrared luminosity function they derive is comparable to that of the IRAS bright galaxy sample (Soifer et al 1987), From this they conclude that about $1 \%$ of all IRAS bright galaxies are in compact groups. This agrees with the observation that 3 of the 324 galaxies in the Soifer et al sample are in fact in Hickson's compact group catalog.

d) X-ray

Very little X-ray data is available for compact groups. Bahcall, Harris and Rood (1984) observed five HCGs with the Einstein observatory. Three of these were detected and two showed diffuse soft X-ray emission typical of hot intracluster gas. Hickson et al (1989) searched data from the HEAO 1 A-2 experiment (Rothschild et al 1979) for counts in the fields of the HCGs. However, it was possible only to place an upper limit on the $2-10 \mathrm{keV}$ luminosity, which is consistent with the Einstein results.

\section{Discordant Redshifts}

The problem of discordant redshifts in compact groups remains unresolved after many years. The key question is whether discordant redshifts in compact groups can be explained by chance superpositions of unrelated galaxies near the line of sight. Arguments against this hypothesis have been advanced by Burbidge and Burbidge (1961), Arp (1973), Arp and Lorre (1976), Sulentic (1983), Sulentic and Arp (1983), Sulentic and Lorre (1983) among others. Studies supporting the superposition hypothesis have been published by Rose (1977) and Hickson, Kindl and Huchra (1988a). Two basic arguments have been raised against the hypothesis: 1) that there is evidence of physical association between discordant and accordant redshift galaxies and 2) that the required chance superpositions are unlikely. The first argument remains controversial, neither side having convinced the other. The second argument can be examined by statistical analysis.

The most recent work is that of Hickson, Kindl and Huchra (1988a) who use the homogeneity of the HCG sample to estimate the number of discordant redshift quintets expected by chance superposition. They predict that about $35 \%$ of all compact quintets should contain one galaxy with a discordant (more than $1000 \mathrm{~km} \mathrm{~s}^{-1}$ from the mean) redshift. Although the redshift measurements for this sample are not yet complete, this figure is in agreement with the observation that 4 of the 10 quintets with complete redshifts have 
one discordant redshift. From this it would appear that there is no compelling reason to believe that the number of groups with discordant redshifts is significantly greater than the number expected from chance superpositions. However, the case is not yet closed. It will be interesting to see if this prediction still holds when complete redshifts are obtained for the full sample of 25 quintets. In addition, the observed discordant-redshift galaxies show a strong preference for central location, which would not be expected from chance superpositions. A possible explanation for this effect could come from gravitational lensing (Hammar and Notale 1986). Amplification of the light from background passing through the gravitational potential of the group would increase the probability of finding a superposed background galaxy near the center of the mass distribution of the group. It should be possible to test this hypothesis statistically with the HCG sample when the redshift survey is complete. If gravitational lensing is a significant factor, it may even be possible to obtain mass estimates for the groups independent of the virial theorem.

\section{Physical Nature}

Also controversial is the nature of the groups themselves. A non-detection of background light in two groups led Rose (1979) to conclude that "the most plausible explanation for compact groups is that they are temporary unbound configurations forming within loose groups". This explanation was rejected by Mamon (1986) who concluded that most HCGs are "chance alignments of galaxies within loose groups", and therefore are not physically dense.

Hickson and Rood (1988) summarized the observational evidence for high group density, and concluded, from a Monte-Carlo computation of alignment probabilities, that the probability of forming the HCGs by chance alignment is very small. Walke and Mamon (1989, see also Mamon's paper in these proceedings) obtained similar probability estimates, but argued that the richest loose groups have a significant chance of having compact subgroups from chance alignments. They concluded that "roughly half of Hickson's compact groups are chance alignments of galaxies within intermediate density groups and small clusters". This explanation, however, requires that almost all compact groups should occur in clusters. Walke and Mamon predict that 6 times as many compact configurations will occur in the Virgo cluster than in the entire Tully (1987) loose group catalog. This seems to be inconsistent with the observation that hardly any of the HCGs are found in clusters.

One should not conclude that there is no contamination of compact groups with unrelated galaxies. Indeed, the contamination is likely to be significant. From the environment studies describe above, Hickson (1990, unpublished) estimates that about $13 \%$ of galaxies in HCGs are superposed members of a loose group, and another $17 \%$ are superposed field galaxies with a discordant redshift. Thus perhaps $30 \%$ of the galaxies in the HCGs are superpositions. 


\section{Dynamics}

The redshift survey of Hickson and Huchra (1990) reveals a wide range of radial velocity dispersions in the HCGs, with a median of about $200 \mathrm{~km} \mathrm{sec}^{-1}$. The median projected separation between galaxies is about $40 \mathrm{~h}^{-1} \mathrm{kpc}$, and the median mass-to-light ratio, estimated from the virial theorem is about $40 \mathrm{~h} M_{\odot} / L_{\odot}$. This is larger than typical mass-to-light ratio of typical galaxies by almost an order of magnitude. Either the groups are unbound (Ambartsumyan 1958) or they contain a substantial amount of dark matter. The conventional picture (eg. Barnes 1985, 1989, 1990) is that compact groups evolve from looser groups and will be destroyed by mergers on a relatively short timescale. Estimates of the typical lifetime of compact groups vary, but a reasonable estimate is of order a tenth of the age of the universe (White 1990). Assuming that the present epoch is in no way special, one expects as many as ten merger products of compact groups for every compact group seen today. Many studies suggest that these relics would resemble elliptical galaxies. Thus as much as $10 \%$ of the light of the total galaxy population may result from relics of compact groups. This figure is probably an upper limit as several effects can reduce it. The presence of substantial dark matter in the groups increases the dynamical friction timescale, increasing the lifetimes. Also, the luminosity density of compact groups may be overestimated because of brightening of the galaxies due to star formation triggered by interactions (this would increase both the number of detected groups and the mean luminosity per group). Certainly, this number is not inconsistent with Schechter and Dressler's(1987) estimate that at least $15 \%$ of the luminosity density comes from elliptical galaxies. Of course it remains to be demonstrated that the relics would have the required properties. Further observations, many of which are now in progress, combined with increasingly more sophisticated dynamical studies, should elucidate the role of compact groups in the formation and evolution of galaxies.

\section{Acknowledgements}

I thank Kochu Menon and Claudia Mendes de Oliveira for helpful discussions and comments on the manuscript. This work was supported by a grant from the Natural Sciences and Engineering Research Council of Canada.

\section{References}

Ambartsumyan, V. A. 1958, Izv. Acad. Nauk. Arm. SSR, Fiz.-Mat., 11, 9.

Ambartsumyan, V. A. 1975, Astrofiz., 10, 471.

Amirkhanyan, A. S., and Egikyan, A. G 1987, Astrofiz.27, .

Arkhipova, V. P. 1982 Astron. Zh., 59, 202.

Arkhipova, V. P., Zasov, A. V., Noskova, R. I., and Sil'chenko, O. K. 1987 Astron. Zh., 64, 
1161.

Arp, H. C. 1966, Ap. J. Suppl., 14, 1.

Arp, H. C. $1973, A p . J ., ~ 183,411$.

Arp, H. C., and Lorre J. J. 1976, Ap. J., 210, 58.

Arp, H. C., Burbidge, G. R., and Jones, T. W. 1973, P. A. S. P., 85, 423.

Bahcall, N. A., Harris, D. E., and Rood, H. J. 1984, Ap. J. (Letters), 284, L29.

Baier, F. W., Petrosyan, M. B., Tiersch, H., and Shakhbazyan, R. K. 1974, Astrofiz., 10, 327.

Baier, F. W., Tiersch, H. 1975, Astrofiz., 11, 221.

Baier, F. W., Tiersch, H. 1976a, Astrofiz., 12, 7.

Baier, F. W., Tiersch, H. 1976b, Astrofiz., 12, 409.

Baier, F. W., Tiersch, H. 1978, Astrofiz., 14, 279.

Baier, F. W., Tiersch, H. 1979, Astrofiz., 15, 33.

Barbieri, C. , Casini, C. , Jeidmann, J., di Serego, S., and Zambon, M. 1979, Astr. Ap. Suppl., 37, 559.

Barnes, J. 1985, M. N. R. A. S., 215, 517.

Barnes, J. 1989, Nature, 338, 123.

Barnes, J. 1990, to appear in Dynamics and Interactions of Galaxies, ed. R. Wielen (Heidelberg: Springer-Verlag).

Beekman, G. W. 1974, Zenit, 1, 24.

Bingelli, B., Sandage, A., and Tamman, G. A. 1988, Ann. Rev. Astr. Ap., 26, 509.

Börngen, F., and Kalloglyan, A. T. 1974, Astrofiz., 10, 21.

Burbidge, E. M. and Burbidge, G. R. 1961, Ap. J., 134, 244.

Burbidge, E. M. and Sargent, W. L. W. 1971, Ap. J., in Nuclei of Galaxies, ed. D. J. K. O'Connell (Amsterdam: North-Holland).

Hammar, F. and Nottale, L. 1986, Astr. Ap., 155, 420.

Heiligman, G. M., and Turner, E. L 1980, Ap. J., 236, 745. 
Hickson, P. 1982, Ap. J., 255, 382.

Hickson, P., and Huchra, J. P. 1990, in preparation.

Hickson, P., Kindl, E., Auman, J. R. 1989, Ap. J. Suppl., 70, 687.

Hickson, P., Menon, T. K., Palumbo, G. G. C., and Persic, M. 1988, Ap. J., 341, 679.

Hickson, P., Ninkov, Z., Huchra, J., and Mamon G. 1984, in Clusters and Groups of Galaxies, ed F. Mardirossian, G. Giuricin, and M. Mezzetti (Dordrecht: Reidel), p. 367.

Hickson. P., Richstone, D. O., and Turner, E. L. 1977, Ap. J., 213, 323.

Hickson, P., Kindl, E., and Auman, J. R. 1989, Ap. J. Suppl., 70, 687.

Hickson, P., Kindl, E., and Huchra, J. P. 1988a, Ap. J. (Letters), 329, L65.

Hickson, P., Kindl, E., and Huchra, J. P. 1988b, Ap. J., 331, 64 .

Hickson, P., and Rood, H. J. 1988, Ap. J., 331, L69.

Kindl, E. 1990, Ph. D. Thesis, University of British Columbia.

Kirshner, R. P., and Malumuth, E. M. 1980, Ap. J.,236, 366.

Klimek 1974, Urania Kraków, 45, 34.

Kodaira, Iye, Okamura, and Stockton 1988, Pub. Astr. Soc. Japan, 40, 533.

Massey, P. 1977, P. A. S. P., 89, 13.

Malykh, S. A., and Orlov, V. V. 1986. Astrofiz.. 24, 445.

Mamon, G. A. 1987, Ap. J., 321, 622.

Menon, T. K., and Hickson, P. 1985, Ap. J., 296, 60.

Mirzoyan, L. V., Miller, J. S., and Osterbrock, D. E. 1975, Ap. J., 196, 687.

Palumbo, G. G. C., Tornatore, V., Baiesi-Pillastrini, G., Hickson, P., and Mendes de Oliveira, C. 1990 , in preparation.

Petrosyan, M. B. 1974, Astrofiz., 10, 471.

Petrosyan, M. B. 1978, Astrofiz., 14, 631.

Robinson, L. B., and Wampler, E. J. 1973, Ap. J. (Letters), 179, L135.

Rood, H. J., and Williams, B. A.. 1989, Ap. J., 339, 772.

Rose J. A. 1977, Ap. J., 211, 311. 
Rose J. A. 1979, Ap. J., 231, 10.

Rothschild, R., et al 1979, Space Sci. Instrum., 4, 269.

Rubin, V. C., Hunter, D., and Ford. W. K., Jr. 1990, in preparation.

Seyfert, C. K. 1948a, Phys. Rev., 74, 129.

Seyfert, C. K. 1948b, A. J., 53, 203.

Schechter, P. L., and Dressler, A. 1987, A. J., 94, 563.

Shakhbazyan, R. K. 1957, Astron. Tsirk., 177, 11.

Shakhbazyan, R. K. 1973, Astrofiz., 9, 495.

Shakhbazyan, R. K. 1978, Astrofiz., 14, 273.

Shakhbazyan, R. K., and Amirkhanyan 1978, Astrofiz., 14, 455.

Shakhbazyan, R. K. and Petrosyan 1974, Astrofiz., 10, 13.

Soifer, B. T., Sanders, D. B., Madore, B. F., Neugebauer, G., Danielson, G. E., Elias, J. H., Lonsdale, C. J., and Rice, W. L., 1987, Ap. J., 320, 238.

Stephan, M. E. 1877, M. N. R. A. S., 37, 334.

Sulentic, J. W. 1983, Ap. J., 270, 417.

Sulentic, J. W. 1987, Ap. J., 322, 605.

Sulentic, J. W., and Arp, H. C. 1983, A. J., 88, 267.

Sulentic, J. W., and Lorre, J. J. 1983, Astr. Ap., 120, 36.

Thompson, L. A. 1976, P. A. S. P., 88, 662 .

Tiersch, H. 1976, Astron. Nachr., 297, 301.

Tikhonov, N. A. 1986, Soobshch. Spets. Astrofiz. Obs., 49, 69.

Tikhonov, N. A. 1987a, Soobshch. Spets. Astrofiz. Obs., 52, 51.

Tikhonov, N. A. 1987b, Astrofiz., 27, 253.

Tikhonov, N. A. 1989, private communication.

Tovmasyan, G. M., and Shakhbazyan, É. Ts. 1981, Astrofiz., 17, 265.

Tully, R. B. 1987, Ap. J., 321, 280. 
Turner, E. L., and Sargent W. L. W. 1975, private communication.

Vorontsov-Vel'yaminov, B. A., Dostal', V. A., and Metlov, V. G. 1980, Pis'ma Astron. Zh., 6, 394 .

Walke, D. G., and Mamon, G. A. 1989 Astr. Ap., 295, 291.

Wesselink, A. J. 1974, in New Problems in Astrometry, IAU Symposium No. 61, ed. W. Gliese, C. A. Murray, and R. H. Tucker (Dordrecht: Reidel), p. 201.

White, S. D. M. 1990, to appear in Dynamics and Interactions of Galaxies, ed. R. Wielen (Heidelberg: Springer-Verlag).

Whitmore, B. C. 1990, to appear in the Proceedings of the 1989 STScI Workshop, Clusters of Galaxies, (Cambridge: Cambridge University Press).

Williams, B. A., and Rood, H. J. 1987, Ap. J. Suppl., 63, 265.

Williams, B. A., and van Gorkom, J. H. 1988, Ap. J., 287, 66.

Vorontsov-Vel'yaminov, B. A. 1959, Atlas and Catalog of Interacting Galaxies, Vol 1 (Sternberg Institute, Moscow State University, Moscow).

Vorontsov-Vel'yaminov, B. A., Atlas of Interacting Galaxies, Part II, Astr. Ap. Suppl., 28, 1.

\section{DISCUSSION}

Petrosian: Did you look at the relationship of AGN's and emission-line galaxies to HCGs?

Bickson: It is a question for the future. We must have more homogeneous high-resolution spectral observations for all members of HCGs to get the correct answer to this question.

Burbidge: Surely your last point is the most important one. Until you can place limits on the behavior of the IMF in these systems, all of the conclusions concerning star formation and evolution are meaningless.

Rennicutt: The situation is not quite that bad! The agreement in the star formation rates derived from $\overline{\mathrm{Ha}}$, UV, and UBV data, all which trace different parts of the IMF, tells us that the rates cannot be in error by more than factors of a few, and hence the orders-of-magnitude bursts which are observed in many interacting systems cannot be simply artifacts of an unusual IMF. However I wholly agree that our ignorance about the IMF in these galaxies is the main impediment to understanding their detailed evolutionary properties. 


\section{DISCUSSION}

Roberts: The $\mathrm{M} / \mathrm{L}$ value of 40 that you quoted was for the entire sample. Have you computed $M / L^{\prime}$ 's using only those galaxies within groups that show signs of interaction?

Hickson: The value of 40 is the median of all groups with three or more measured velocities. As you know $M / L$ estimators are rather unstable when such small numbers of galaxies are involved. I am hesitant to consider subdivision of the sample until we have finished measuring redshifts for all of the galaxies.

Zasov: A small comment about nests. We have observed spectroscopically at least a dozen nests and related objects. In most cases a nest is in reality a single object with clumpy structure without any hint to be a merger. I wonder if the nests also follow dependencies that you obtained for the other compact systems.

Hickson: I think that the Vorontsov-Vel'yaminov nests are rather different from the compact groups that I described. The components of nests are not clearly recognizable as spiral or elliptical galaxies. As you say, these objects may be single galaxies, in general. It is possible that a few of the groups in my catalog (No. 18, for example) may be single irregular galaxies, but this would be a very small minority.

Appleton: Your plot of spiral fraction versus velocity dispersion is interesting. As the velocity dispersion goes up, the spiral fraction goes down. Are the spirals replaced with giant ellipticals, dwarf ellipticals, or irregulars.?

Hickson: In calculating spiral fraction, I group blue irregular galaxies with spiral galaxies. In the higher velocity dispersion groups, the spiral galaxies are replaced by elliptical galaxies in general. These are not dwarf ellipticals, but have typical absolute blue magnitudes of order -20 .

Hutchings: Can you make a statistical test on discordant velocities on the basis of whether they are foreground or background?

Hickson: In most cases, the discordant galaxies have higher redshifts that the other group members, smaller sizes, and fainter magnitudes. Those with lower redshifts tend to be larger and brighter, as you would expect if their redshifts are cosmological. I haven't tried to quantify this, but it might lead to interesting results.

Navarro: Could you comment on J. Barnes simulations of the dynamical evolution of a compact group, especially on his suggestion that compact groups might be the final product of the dynamical evolution of loose groups. 
Hickson: I think that the general picture of loose groups being progenitors of compact groups is probably correct, although one has to look carefully at the lifetimes and relative numbers. It would seem likely that compact groups could form from bound subcondensations in loose groups, by dynamical friction. Barnes' simulations beautifully illustrate the evolution of such systems, once they are already quite dense, but many questions remain to be answered. The dark matter, for example, is very important. At what point does it detach from individual galaxy halos and become distributed throughout the group?

Huradian: What can you say about the possible rotation of the dense groups? Is there direct or indirect evidence for the rotation of such groups as a whole?

Hickson: I do not find any systematic trends in the galaxy velocities that could be attributed to rotation of the groups. However, the HI observations of Williams and van Gorkom do show systematic rotation of the large HI clouds enveloping the groups that they studied.

Forbes: What do the X-ray observations of the Hickson groups show?

Hickson: The only positive detection of $\mathrm{X}$-ray emission from compact groups is reported by Bahcall, Harris and Rood (1984). They detected three of five groups (not all HCGs) with the Einstein observatory. In two of these groups, stephan's quintet and $\operatorname{Arp} 330$, extended soft $X-r a y$ emission was found which is likely caused by hot intracluster gas. They conclude that the $\mathrm{X}$ ray luminosities and temperatures are consistent and extrapolation from richer groups and clusters. In the third detected group (Arp 318) the X-ray emission may originate in the member galaxies.

Mamon: You mentioned that 308 of the galaxies in your compact groups are morphologically disturbed. What is the fraction of groups that contain at least 3 morphologically disturbed galaxies (thus showing groups with more than just one interacting binary)?

Hickson: About one third of all groups.

Khachikian: What do you mean when you say that there are not enough faint galaxies in the groups?

Hickson: Individual groups have typically four or five bright galaxies, but relatively few faint ones. Whereas, in a random sample of field galaxies there are more faint galaxies than bright ones. Since the overall luminosity of the compact group galaxies appears to be similar to that of the field, this observation implies that there is luminosity concordance in the groups. In other words, there are groups of bright galaxies, and groups of faint galaxies. This may be a result of the selection criteria, or it may be due to physical processes. 